\title{
The contradiction of breast implant illness
}

\author{
Eduardo de Faria Castro Fleury^ \\ Instituto Brasileiro de Controle do Câncer Oncologia (IBCC Oncologia), São Paulo, Brazil \\ Correspondence to: Eduardo de Faria Castro Fleury, MD, PhD. Rua Maestro Chiaffarelli, 409 Jardim Paulista, São Paulo, Brazil. \\ Email: edufleury@hotmail.com. \\ Response to: Kaplan J, Rohrich R. Breast implant illness: a topic in review. Gland Surg 2021;10:430-43.
}

Submitted Mar 04, 2021. Accepted for publication May 09, 2021.

doi: $10.21037 /$ gs-21-135

View this article at: https://dx.doi.org/10.21037/gs-21-135

We read with great interest the recent article entitled "Breast implant illness: a topic in review" published by J. Kaplan and R. Rohrich (1). The authors aimed to discuss breast implant illness (BII) and breast implant safety. The authors performed a bibliographic review of manuscripts available in the medical literature and concluded that there are no concrete or evidence-based studies that support a new syndrome described as BII. However, when reading the well-written and referenced article, two additional points could be added to improve the manuscript discussion.

The first point is related to time. The article published in the February 2021 edition of Gland Surgery journal was submitted in February 2020 and accepted for publication in July 2020. During the article's acceptance and publication period, the Food and Drug Administration (FDA) agency published recommendations regarding silicone breast implants on September 29, 2020, with the title "Breast Implants - certain labeling recommendations to improve patient communication" (2). The recommendations published by the FDA article are relevant to advise caution, follow-up, and management for patients with breast implants. Unfortunately, these recommendations could not be part of this critical review carried out by the authors due to publication time issues.

The FDA recommends using a patient booklet/ brochure and a device card of the patient labeling for breast implants. The booklet/brochure should include a boxed warning, patient decision checklist, rupture screening recommendations, materials/device description, and other patient information. The FDA suggested boxed warning is: * "Breast implants are not considered lifetime devices. The longer people have them, the greater the chances are that they will develop complications, some of which will require more surgery."

* "Breast implants have been associated with the development of a cancer of the immune system called breast implantassociated anaplastic large cell lymphoma (BIA-ALCL). This cancer occurs more commonly in patients with textured breast implants than smooth implants, although rates are not well defined. Some patients have died from BIA-ALCL."

* "Patients receiving breast implants have reported a variety of systemic symptoms such as joint pain, muscle aches, confusion, chronic fatigue, autoimmune diseases and others. Individual patient risk for developing these symptoms has not been well established. Some patients report complete resolution of symptoms when the implants are removed without replacement."

These recommendations were derived from the 2019 Panel meeting, where patients and panel members expressed concern about not knowing the materials used in breast implants and the possible deleterious health effects of these materials. We believe that the information in this new FDA recommendation would be of fundamental importance in discussing the contradiction for the current article statements.

The second point, on the other hand, consists of the gel bleeding/shedding concept. The authors do not address in the manuscript the complications potential resulting from local/systemic toxicity and exposure to silicone particles, especially the polydimethylsiloxane (PDMS 4). According to the FDA recommendation, patients should be advised that: "I understand that gel bleed (small quantities of chemicals diffusing from the implant shell) of silicone gel-filled implants

$\wedge$ ORCID: 0000-0002-5334-7134. 
may occur." (2).

Since the year 2017, we have carried out a research protocol regarding silicone implants' complications in patients referred to breast magnetic resonance imaging (BMRI) in our service. Our prospective study evaluated about 3,000 patients with silicone implants. We observed common image findings in symptomatic patients referred to BMRI and described these findings as silicone-induced granuloma of breast implant capsule (SIGBIC). When correlating the imaging findings with histology, we observed that the fibrous capsules masses contained silicone particles associated with inflammatory cells such as lymphocytes and foamy macrophages. In 2018, we published a review article where we discussed the pathophysiology of SIGBIC and another article where we described the main imaging findings for SIGBIC diagnosis $(3,4)$. We found that our patients' clinical complaints were similar to those described by autoimmune syndrome induced by adjuvants (ASIA) syndrome $(3,4)$. In 2020, we published an article in which we presented the presence of SIGBIC in $30.6 \%$ patients referred to diagnostic BMRI scans (5).

We have also published a case report in which we described a patient with BIA-ALCL in one breast and SIGBIC in the contralateral breast (6). When microscopically evaluating the implant shell, permeability loss and shell degradations signs were evident in both implants. The only difference was the voluminous intracapsular collection in the BIA-ALCL side. In another published article, we showed an inflammatory reaction of the fibrous capsule, where the differential diagnosis of SIGBIC and BIA-ALCL was raised (7). Finally, in a recent article from 2021, we presented two cases of patients with pericapsular breast carcinoma, where the tumor appears in the implant's shell degradation area, with direct and chronic exposure of the silicone's internal content. Based on an index case, we proposed the direct and continuous silicone exposure's carcinogenic potential pathway (8). We also have evidence of silicone migration to distant organs.

According to the authors in the current article, they stated: "Plastic surgeons, like all physicians, took a Hippocratic oath to 'do no harm' and have a responsibility to best inform their patients on the safety of these devices and to listen with a kind ear when patients present with symptoms and complaints that bave the potential to be associated with silicone breast implants." Finally, the authors conclude: "The purpose of this review article is to discuss the current state of scientific evidence related to the safety of silicone breast implants. In times of uncertainty, unwanted noise can easily distort research-based evidence. It is the responsibility of all physicians, especially plastic surgeons, to always put patient safety first and to critically self-evaluate our practice and the industry partners who serve our patients. Few medical devices have undergone the degree of scrutiny and speculation as silicone breast implants."

Our group is in full agreement with the authors of the doctors' responsibility for the patients' health. The contradiction sustains science. The contradiction makes science evolve and improve patient care in a dynamic way. Based on the two points highlighted in this letter to the editor and our published manuscripts, we believe that BII debate should be intensified. We consider BII as a spectrum of diseases with a common trigger point in our five-year research experience: gel bleeding; and the disease's severity could range from capsular contracture and systemic symptoms to more local aggressive manifestations like BIAALCL and breast carcinoma. The patient's voices suffered from BII should not be silenced by science.

\section{Acknowledgments}

Funding: None.

\section{Footnote}

Provenance and Peer Review: This article was a standard submission to the journal. The article did not undergo external peer review.

Conflicts of Interest: The author has completed the ICMJE uniform disclosure form (available at https://dx.doi. org/10.21037/gs-21-135). The author has no conflicts of interest to declare.

Ethical Statement: The author is accountable for all aspects of the work in ensuring that questions related to the accuracy or integrity of any part of the work are appropriately investigated and resolved.

Open Access Statement: This is an Open Access article distributed in accordance with the Creative Commons Attribution-NonCommercial-NoDerivs 4.0 International License (CC BY-NC-ND 4.0), which permits the noncommercial replication and distribution of the article with the strict proviso that no changes or edits are made and the original work is properly cited (including links to both the formal publication through the relevant DOI and the license). See: https://creativecommons.org/licenses/by-nc-nd/4.0/. 


\section{References}

1. Kaplan J, Rohrich R. Breast implant illness: a topic in review. Gland Surg 2021;10:430-43.

2. Food and Drug Administration. Breast Implants Certain Labeling Recommendations to Improve Patient Communication: Guidance for Industry and Food and Drug Administration Staff (fda.gov). Accessed 25 February 2021. Available online: https://www.fda.gov/ media/131885/download

3. de Faria Castro Fleury E, D'Alessandro GS, Lordelo Wludarski SC. Silicone-Induced Granuloma of Breast Implant Capsule (SIGBIC): Histopathology and Radiological Correlation. J Immunol Res 2018;2018:6784971.

4. de Faria Castro Fleury E, Gianini AC, Ayres V, et al. Breast magnetic resonance imaging: tips for the diagnosis

Cite this article as: Fleury EFC. The contradiction of breast implant illness. Gland Surg 2021;10(6):2081-2083. doi: 10.21037/ gs-21-135 of silicone-induced granuloma of a breast implant capsule (SIGBIC). Insights Imaging 2017;8:439-46.

5. Fleury EFC. Silicone Induced Granuloma of Breast Implant Capsule (SIGBIC) diagnosis: Breast Magnetic Resonance (BMR) sensitivity to detect silicone bleeding. PLoS One 2020;15:e0235050.

6. Fleury EFC. Synchronous breast implant associated anaplastic large cell lymphoma (BIA-ALCL) and silicone induced granuloma of breast implant capsule (SIGBIC): What to learn. Radiol Case Rep 2020;15:1736-42.

7. Castro C, Fernandes D, Mendonça M, et al. Siliconeinduced granuloma of breast implant capsule mimicking anaplastic large cell lymphoma. Breast J 2020;26:1028-30.

8. Fleury E, Nimir C, D'Alessandro GS. The Breast Tumor Microenvironment: Could Silicone Breast Implant Elicit Breast Carcinoma? Breast Cancer (Dove Med Press) 2021;13:45-58. 